\title{
Pain Management and Anesthesia in Total Knee Arthroplasty
}

\author{
Sang Jun Song, MD, PhD \\ Department of Orthopaedic Surgery, Kyung Hee University College of Medicine, Seoul, Korea
}

Pain is one of the major concerns for patients contemplating total knee arthroplasty (TKA). Appropriate pain management improves patient satisfaction ${ }^{1)}$, promotes functional recovery, and reduces the risk of postoperative complications ${ }^{2}$. Pain management has been advanced with the principle of preemptive analgesia and use of multimodal approaches ${ }^{3)}$. The clinical practice guidelines for pain management after TKA were approved by Korean Knee Society on February, 2012, and eleven recommendations were developed based on a systematic review of research evidence ${ }^{4)}$. However, the efficacy and safety of various protocols are still controversial ${ }^{1,5,6)}$. The theme of this issue of the Knee Surgery \& Related Research (KSRR) is pain management and anesthesia in TKA. The current issue contains three articles including two review articles and one original article on this theme. One review article by Lee described the general concept of TKA pain and dealt with the current concept of periarticular injections and peripheral nerve blocks. Other review article by In et al. focused on a recently emerging but relatively controversial topic on the efficacy and safety of femoral nerve block and adductor canal block. The last original article compared the occurrences of perioperative complications with staying time in the operation room between general and spinal anesthesia for primary TKA. In addition, one recent original article in $K S R R^{7}$ is worth considering together. It compared the analgesic effect of periarticular injections

Received May 10, 2017; Accepted May 10, 2017

Correspondence to: Sang Jun Song, MD, PhD

Department of Orthopaedic Surgery, Kyung Hee University College of Medicine, 23 Kyunghee-daero, Dongdaemun-gu, Seoul 02447, Korea

Tel: +82-2-958-9489, Fax: +82-2-964-3865

E-mail: songsjun@khmc.or.kr

This is an Open Access article distributed under the terms of the Creative Commons Attribution Non-Commercial License (http://creativecommons.org/licenses/by-nc/4.0/) which permits unrestricted non-commercial use, distribution, and reproduction in any medium, provided the original work is properly cited. and femoral nerve block.

Pain management protocols in TKA include oral or parenteral analgesics, patient-controlled analgesia, periarticular injections, peripheral nerve blocks, and continuous epidural anesthesia ${ }^{1,8)}$. Periarticular injections and peripheral nerve blocks are particularly popular modalities because of sufficient evidence of advantages and less adverse effects than systemic analgesics ${ }^{9)}$.

The proper dosage and composition of periarticular injections have not been established ${ }^{1,2)}$. Time-dependent efficacy and rebound pain are weak points of this modality ${ }^{10)}$. For the composition of a periarticular injection, additional drugs besides major local anesthetic and analgesic agents (bupivacaine, ropivacaine, ketololac, and clonidine) include epinephrine and steroids for lengthening the duration of action ${ }^{11,12}$. Lee reviewed randomized controlled trials on the synergistic effects of multimodal regimens for periarticular injections. The advantages and disadvantages of liposomal bupivacaine and additional steroids were fairly described.

Because of excellent pain relief and opioid sparing effect, a peripheral nerve block is commonly used among multiple analgesic modalities ${ }^{4}$. The target peripheral nerves can be femoral, sciatic, and saphenous nerves ${ }^{13)}$. The procedure is performed with a single or continuous infusion ${ }^{1,6}$. Caution has been advised with the use of a continuous femoral nerve block because it has been associated with quadriceps femoris muscle weakness, increased risk of falling after TKA, and compromised proprioception such as femoral neuritis ${ }^{4,6}$. Therefore, although femoral nerve block has been considered as the standard anesthetic technique for its efficacy, adduction canal block is regarded as a reasonable alternative for quadriceps strength preservation ${ }^{4}$. In et al. performed a comprehensive review of the anatomy, infiltration techniques, analgesic efficacy, and functional recovery after the adductor canal block, and compared the analgesic efficacy and functional re- 
covery of the femoral nerve block and adductor canal block from the previous literatures. The article summarizes useful information on the current concept of the peripheral nerve block for pain management after TKA. Detailed remarks on ultrasonography images with schematic drawings of the anatomical structures will provide a comprehensive understanding of the adductor canal block.

The analgesic efficacy and safety of periarticular injections and femoral nerve block still remain controversial ${ }^{14,15)}$. Lee reviewed recent meta-analyses on this topic with careful consideration into administration methods. One recent article on this topic was also published in KSRR by Nakagawa et al. ${ }^{7}$. They compared the amount of additionally used analgesics between a periarticular injection and a single-shot femoral nerve block after TKA. Their conclusion was that the analgesic effect of the periarticular injection was greater than that of the single-shot femoral nerve block without serious complications. However, controversy still surrounds this issue due to the huge heterogeneity of dosage, analgesics regimens, administration methods, and techniques in previous literatures ${ }^{6,14,15)}$.

It is generally known that regional anesthesia either spinal or epidural has greater advantages than general anesthesia, in the absence of specific contraindications such as coagulopathy and risk of epidural hematoma and paraplegia following hemorrhage $^{4)}$. Regional anesthesia lowers systolic pressure, reducing intraoperative bleeding, frequency of nausea and vomiting, and pulmonary and cardiovascular complications ${ }^{4}$. Yim et al. compared the occurrences of perioperative complications and staying time in the operation room between spinal and general anesthesia for primary TKA. Spinal anesthesia decreased preoperative and postoperative staying times in the operation room, hospitalization duration, transfusion rates, and surgical site infection rates. They could have supposed that the selection of anesthetic methods is based on the various considering factors including the advantages of postoperative analgesic effect and rapid functional recovery after TKA although they focused on the short-term complications.

The current issue has mainly focused on the theme of pain management in TKA to discuss its importance and effect of multiple modalities. All of the articles introduced above are worth generating the interest of the readers of the journal and the need for further related researches.

Many surgeons make constant efforts to reduce pain efficiently with various methods in pre-, intra-, and postoperative periods of TKA. Experts have summarized and presented the current concept of pain management in TKA. Further research on pain management is warranted for the purpose of optimizing protocols and minimizing adverse effects.

\section{References}

1. Dalury DF, Lieberman JR, Macdonald SJ. Current and innovative pain management techniques in total knee arthroplasty. Instr Course Lect. 2012;61:383-8.

2. Parvataneni HK, Ranawat AS, Ranawat CS. The use of local periarticular injections in the management of postoperative pain after total hip and knee replacement: a multimodal approach. Instr Course Lect. 2007;56:125-31.

3. Meftah M, Wong AC, Nawabi DH, Yun RJ, Ranawat AS, Ranawat CS. Pain management after total knee arthroplasty using a multimodal approach. Orthopedics. 2012;35:e660-4.

4. Korean Knee Society. Guidelines for the management of postoperative pain after total knee arthroplasty. Knee Surg Relat Res. 2012;24:201-7.

5. Fan L, Yu X, Zan P, Liu J, Ji T, Li G. Comparison of local infiltration analgesia with femoral nerve block for total knee arthroplasty: a prospective, randomized clinical trial. J Arthroplasty. 2016;31:1361-5.

6. Pagnotto MR, Pagnano MW. Multimodal pain management with peripheral nerve blocks for total knee arthroplasty. Instr Course Lect. 2012;61:389-95.

7. Nakagawa S, Arai Y, Inoue H, Kan H, Hino M, Ichimaru S, Ikoma K, Fujiwara H, Amaya F, Sawa T, Kubo T. Comparative effects of periarticular multimodal drug injection and single-shot femoral nerve block on pain following total knee arthroplasty and factors influencing their effectiveness. Knee Surg Relat Res. 2016;28:233-8.

8. Gwam CU, Mistry JB, Khlopas A, Chughtai M, Thomas M, Mont MA, Delanois RE. Does addition of multimodal periarticular analgesia to adductor canal block improve lengths of stay, pain, discharge status, and opioid use after total knee arthroplasty? J Arthroplasty. 2017;32:1470-3.

9. Kuang MJ, Du Y, Ma JX, He W, Fu L, Ma XL. The efficacy of liposomal bupivacaine using periarticular injection in total knee arthroplasty: a systematic review and meta-analysis. J Arthroplasty. 2017;32:1395-402.

10. Alijanipour P, Tan TL, Matthews CN, Viola JR, Purtill JJ, Rothman RH, Parvizi J, Austin MS. Periarticular injection of liposomal bupivacaine offers no benefit over standard bupivacaine in total knee arthroplasty: a prospective, randomized, controlled trial. J Arthroplasty. 2017;32:628-34.

11. Kelley TC, Adams MJ, Mulliken BD, Dalury DF. Efficacy 
of multimodal perioperative analgesia protocol with periarticular medication injection in total knee arthroplasty: a randomized, double-blinded study. J Arthroplasty. 2013;28: 1274-7.

12. Tran J, Schwarzkopf R. Local infiltration anesthesia with steroids in total knee arthroplasty: A systematic review of randomized control trials. J Orthop. 2015;12(Suppl 1):S4450.

13. Terkawi AS, Mavridis D, Sessler DI, Nunemaker MS, Doais KS, Terkawi RS, Terkawi YS, Petropoulou M, Nemergut EC. Pain management modalities after total knee arthroplasty: a network meta-analysis of 170 randomized controlled trials. Anesthesiology. 2017;126:923-37.

14. Fu H, Wang J, Zhang W, Cheng T, Zhang X. Potential superiority of periarticular injection in analgesic effect and early mobilization ability over femoral nerve block following total knee arthroplasty. Knee Surg Sports Traumatol Arthrosc. 2017;25:291-8.

15. Yu S, Szulc A, Walton S, Bosco J, Iorio R. Pain control and functional milestones in total knee arthroplasty: liposomal bupivacaine versus femoral nerve block. Clin Orthop Relat Res. 2017;475:110-7. 